\section{Public Health Genomics}

Public Health Genomics 2011;14:104-114

DOI: $\underline{10.1159 / 000309852}$
Received: June 24, 2009

Accepted after revision: November 17, 2009

Published online: July 30, 2010

\title{
'Access Arrangements' for Biobanks: A Fine Line between Facilitating and Hindering Collaboration
}

\author{
S. Fortin ${ }^{a} \quad$ S. Pathmasiri ${ }^{a}$ b $\quad$ R. Grintuch ${ }^{b} \quad$ M. Deschênes ${ }^{b, c}$ \\ ${ }^{a}$ Faculty of Law, ${ }^{b}$ Public Population Project in Genomics, and ${ }^{\mathrm{C} C e n t r e}$ de recherche en droit public, \\ University of Montreal, Montreal, Que., Canada
}

\section{Key Words}

Access - Arrangements - Biobanks $\cdot$ Collaboration · Intellectual property $\cdot \mathrm{P}^{3} \mathrm{G} \cdot$ Policies $\cdot$ Procedures $\cdot$ Public Population Project in Genomics • Use

\begin{abstract}
This decade is witnessing the proliferation of large-scale population-based biobanks. Many biobanks have reached the stage of offering access to their collection of data and samples to the scientific community. This, however, requires that access arrangements be established to govern the relationship between biobanks and users. Access arrangements capture the convergence of all normative elements in the life cycle of a biobank: policies, laws, common practices, commitments made by the biobank to participants, the expectations of funders, and the needs of the scientific community. Furthermore, access arrangements shape new legal agreements between 'biobankers' and researchers to ensure appropriate, regulated and efficient use of biobank materials. This paper begins by examining the particularities of access arrangements, identifying the key elements of these new regulatory instruments. Second, the paper looks at various strategies used by biobanks to regulate access and surveys the underlying motivations of these strategies and the impact they can have on potential international collaboration.
\end{abstract}

Third, an example of the challenges encountered in creating access policy is illustrated using the case of CARTaGENE, a biobank based in Montreal, Canada. Last, the paper presents how Public Population Project in Genomics $\left(\mathrm{P}^{3} \mathrm{G}\right)$ facilitates the work of biobankers and improves collaboration throughout the international human genomics research community.

Copyright $\odot 2010$ S. Karger AG, Basel

\section{Introduction}

This decade is witnessing the proliferation of largescale population-based biobanks. The Public Population Project in Genomics $\left(\mathrm{P}^{3} \mathrm{G}\right)$ Observatory website identifies over 146 large-scale population-based studies in genomics with more than 10,000 participants [1]. This new generation of biobanks offers long-term collection options for multiple research uses, with samples often linked to multiple sources of data that can be updated. Many challenges are confronted when setting up such an infrastructure, such as securing support and long-term funding, elaborating innovative scientific protocols, recruiting participants, and developing a governance framework. Many biobanks are at the stage of offering access to data and samples to the scientific community and are therefore officially 'open for business.' This is a crucial step in

\section{KARGER \\ Fax +4161306 1234 \\ E-Mail karger@karger.ch}

www.karger.com
(C) 2010 S. Karger AG, Basel

$1662-4246 / 11 / 0142-0104 \$ 38.00 / 0$

Accessible online at:

www.karger.com/phg
Mylène Deschênes

$\mathrm{P}^{3} \mathrm{G}$ Consortium

3333 Queen Mary Road, Suite 590

Montreal, QC H3V 1A2 (Canada)

Tel. +1 5143437001 ext. 4909, Fax +1 514343 2270, E-Mail mdeschenes@p3g.org 
the life cycle of a biobank and requires that biobank leaders revisit or create rules, procedures and tools to manage the use of their 'data'. For the purpose of this paper, 'data' will refer to all biological material, personal information and research data made available by biobanks to researchers.

Access arrangements capture the convergence of the normative elements surrounding the life cycle of a biobank: policies, laws, commitments towards participants, common practices, expectations of funders and the needs of the scientific community, and assemble in a single location the obligations owing to and owed by the biobank. The Organisation for Economic Co-operation and Development (OECD) defines access arrangements as 'the regulatory, policy and procedural framework established by research institutions, research funding agencies and other partners involved, to determine the conditions of access to and use of research data' [2]. Drawing on the OECD definition, the authors use the term 'access arrangements' to describe all access-related documents, such as policies, procedures, data and material transfer agreements, and other relevant access documents. Access arrangements are, at the same time, the culmination of previous agreements as well as the origin of new contracts to address new circumstances and obligations that arise.

The central challenge in establishing access arrangements is striking an appropriate balance between protecting the biobank (and its volunteer donors) and making it available to researchers. Determining the biobank's optimal use and long-term viability requires a consideration of the motivations that led to its creation.

The first part of this paper identifies key elements pertaining to access arrangements. The paper then turns to an analysis of the various strategies used by biobanks to regulate access and the motivations behind those strategies as well as their impact on potential international collaboration. The objective of this second part is to highlight the strategies employed by biobanks and to flag those which could present limitations to collaboration and the optimal use of data. The interplay between these access strategies and motivations for creating biobanks could probably lead to an interesting follow-up paper in the future. The third section describes the first-hand experience of drafting contracts and policies governing access to data for the CARTaGENE biobank. The paper concludes with the steps undertaken by $\mathrm{P}^{3} \mathrm{G}$ to facilitate the work of biobankers and improve harmonization and collaboration throughout the international scientific community.

Promoting Access to Biobanks

\section{Methods}

Research Design and Scope

The authors of this paper conducted a comparative analysis of publicly available information on access arrangements from the websites of large-scale biobanks. In this section, we describe the scope and parameters of this analysis. The research was carried out from June 2008 to October 2008 and was based on studying access arrangements used in 21 large-scale population-based projects in North America, Europe and Asia, and selected funding agencies in Canada, the United States and the United Kingdom. The list of biobank websites consulted, and a comparative chart displaying the research results, is available online on the $\mathrm{P}^{3} \mathrm{G}$ Observatory website.

First, while the authors of this paper attempted to ensure a broad geographic range that could assess similarities and/or differences in how access arrangements were used and implemented around the world, they were limited to using documentation that was available in English and/or French (either in the original or a translated version). It is worth noting that the language(s) of access arrangements may constitute the first barrier to access for prospective international researchers and collaborators.

Second, the documents used were culled from online resources because they are typically the initial entry point for researchers looking for information on how to access a biobank's collection. The authors of this paper acknowledge the limits to the methodology employed and therefore do not assert that this paper presents a comprehensive picture of access arrangements.

Various access arrangements are referred to throughout this paper as examples of different approaches to access. These examples must be seen as 'potential trends' and not as unique cases. A further note is that the legislative and normative underpinnings of access strategies have not been analyzed since these have been extensively reviewed in ethics and law publications $[3,4]$.

This paper serves as a first step towards developing tools aimed at assisting biobankers in creating access arrangements that will promote the optimal use of their biobank. At this early stage, the authors are aware that current documents, especially those online, are subject to change. However, it is hoped that this compilation of observations will help biobank decision-makers foresee and better understand the choices and the challenges that lie ahead.

\section{Managing Numerous and Diverse Access Requests}

The primary mission of the new generation of largescale biobanks is to offer the scientific community access to a large collection of well-annotated and high-quality data. This is part of the increasingly important trend in science of 'data sharing'. Data sharing is not a mere question of 'openness' but an integral part of fulfilling the biobank's obligations to both funding agencies and participants. The logic is simple: maximizing the availability of resources in turn facilitates and maximizes research. However, the large number of access requests, and inherent complexities of data sharing, requires innovative ap-

Public Health Genomics 2011;14:104-114 
proaches by biobankers. The next section illustrates 4 such approaches: (1) broadening the focus to data sharing, (2) creating an access committee to evaluate requests, (3) using contracts to secure relationships with researcher, and (4) developing a customer service approach.

\section{Data Sharing: The 'Public' Commitment}

In 1996, at the first International Strategy Meeting on Human Genome Sequencing held in Bermuda, scientists unanimously endorsed the Bermuda Statement that argued for a rapid data release policy for DNA sequencing [5]. In 2003, the scientific community reiterated its commitment, emphasizing that data sharing was a tripartite responsibility [6] between resource producers, resource users and funding agencies. In response, many funding agencies and research groups began to promote this approach to data sharing and implemented ways to open access to data from a broad range of publicly funded projects that go beyond sequencing in their funding policies [7].

Since biobanks are mostly publicly funded, the obligation of data sharing is imposed by funders on biobanks. Therefore, access arrangements are in part a reflection of the various approaches to data sharing contained in funding policies.

The funding agencies surveyed here require that beneficiary biobanks implement either a 'data management and data sharing plan' [8], a 'data sharing and data preservation plan' [9] or a 'data release plan' [10] prior to the release of funds. These plans must demonstrate how sharing will increase the significance of their research and benefit society and outline the methods that will be used to share data and results. Funders suggest various strategies to accomplish this, including time-released data, publishing results and placing an emphasis on collaborating with external users.

At the same time, funding agencies also recognize the importance of certain restrictions on data sharing, particularly due to privacy issues. For example, Genome Canada's data sharing policy recommends that biobanks prohibit the transfer of data by researchers to third parties and requires that researchers use data only for research purposes [9]. Accordingly, to limit exclusive access, Genome Canada specifies that 'nondisclosure agreements between sponsors and researchers [for intellectual property reasons] should not be unduly restrictive and must allow publications within a defined and reasonable period of time.' According to the Medical Research Council in the United Kingdom, 'a limited, defined period of exclusive use of data for primary research is reasonable'
[7]. Indeed, the strategies to restrict access suggested by funders often find their way into biobanks' data sharing agreements with researchers. It might be said that the policies of funding agencies can play a decisive role in biobanks' strategies to restrict or promote access to their resources.

\section{Access Committees}

Access committees are an emerging new actor in the field of biobanking. They can either be a specific, independent committee created by the biobank to evaluate access requests or the biobank's pre-existing steering or scientific committee that has been entrusted with new access-related responsibilities.

Access committees evaluate access requests in accordance with the rules or guidelines provided under the access arrangements. They ensure that a careful and appropriate balance is made between protecting the infrastructure and the volunteer participants and maximizing the use of its repository. The committees adjudicate the access arrangements with neutrality, bolstering trust in the administrative process and, after access is granted, enforce rules and conditions of access.

Access committees must develop or be provided with a clear mandate and specific criteria that inform their decision-making process as well as explicit procedures that will allow them to conduct a fair and informed evaluation of all access requests. Furthermore, providing the committee with a well-defined mandate and decisionmaking process will establish and secure the committee's authority and independence.

\section{Complex Contractual Relationships}

Access arrangements are embedded in a biobank's governance framework as an articulation of its concerns and priorities. They are the culmination of a number of agreements established at the creation of the biobank and contain the obligations and conditions included in contracts between users and biobankers. These agreements are struck between multiple actors who, at times, may have divergent interests (e.g. host institutions, funders, users, etc.).

The vehicles generally used to ensure compliance with access conditions are contractual agreements, namely, material transfer agreements (MTAs) and data transfer agreements (DTAs). Depending on the investigator's institutional affiliations, other types of contracts might be used to regulate access. For example, service-level agreements (ALSPAC [11]) and cooperative agreements (within NIH [12]) are contracts geared towards investigators 
Table 1. Summary of potential access strategies employed by biobanks

\begin{tabular}{|c|c|c|c|c|}
\hline Motivation & $\begin{array}{l}\text { Promoting targeted } \\
\text { objectives }\end{array}$ & Promoting the biobank & $\begin{array}{l}\text { Ensuring the highest } \\
\text { standard of research }\end{array}$ & Supporting access \\
\hline Strategy & $\begin{array}{l}\text { Specific evaluation criteria } \\
\text { Collaboration required } \\
\text { Simplified procedures } \\
\quad \text { for internal researchers } \\
\text { Sliding scale costs }\end{array}$ & $\begin{array}{l}\text { Obligation to return results } \\
\text { Acknowledgement and } \\
\text { publication procedures } \\
\text { Legal mechanisms to } \\
\text { resolve potential conflicts }\end{array}$ & $\begin{array}{l}\text { Evaluation of scientific } \\
\quad \text { competence } \\
\text { Periodic reporting } \\
\text { Surveillance mechanisms } \\
\text { Deterrents/penalties }\end{array}$ & $\begin{array}{l}\text { Access available to } \\
\text { different pool of } \\
\text { researchers } \\
\text { Controlled access } \\
\text { costs } \\
\text { Customer service } \\
\text { approach }\end{array}$ \\
\hline
\end{tabular}

whose institutions are affiliated with the biobank and which carry specific costs and conditions that might differ from those in MTAs and DTAs. The parties to the contract are generally the institution which maintains the biobank (university, research center, etc.) and the 'recipient' or 'receiver' and his or her affiliated institution.

In order to access data, the researcher might be required to enter into multiple and distinct contracts. The number of contracts and overlapping requirements can cause confusion about the conditions to which researchers must comply [13].

\section{The Customer Service Approach}

The OECD states that 'access to research data from public funding should be easy, timely, user-friendly and preferably Internet-based' [2]. In response, an emerging trend on biobank websites is a 'customer service approach.' This helps biobanks actively promote and facilitate access to data by guiding researchers through the access 'maze'. For example, some biobanks provide the contact information of a coordinator who can be contacted by the researcher in advance of submitting a request for access. Not only does this provide the researcher with the chance to ask questions or seek assistance in fulfilling specific access requirements, but it allows the opportunity for a 'pre-contact evaluation', which helps reassure the researcher that his or her request is prima facie admissible. Many biobanks also undertake to respond to access requests within a specific timeframe, ranging from a few days to 3 months.

Another emerging approach is the provision of 'immediate' but limited access through a web-based portal. Such access is permitted upon the creation of a passwordprotected user account and acceptance of the terms and conditions. For example, Cancer Care Ontario created iPort, 'a business intelligence tool that enables specific cancer reporting and analysis for the purpose of planning, management and research' [14]. Formal training is required to navigate and use iPort, following an evaluation that determines the level of access to be granted. The level of access granted varies according to the researcher's professional background and the anticipated use of the materials.

\section{The Typology and Impact of Access Strategies}

As described earlier, the many stakeholders involved in the access process are motivated by different (and in some cases divergent) interests. For example, a researcher seeks access to the biobank's data to advance his or her research project, while a biobank seeks to fulfill its operational needs and to regulate the flow of information in and out of the biobank. The former wishes to gain access in the easiest way possible through a transparent evaluation process with clear application steps and at a fair cost. The perspective of biobankers is somewhat more complicated, as demonstrated by the myriad considerations and components factored into creating access arrangements. Biobankers' conceptions of their resource might have evolved or changed since the biobank's inception. Consequently, some initial commitments might become difficult to integrate into the biobank's operations, and other commitments might become obsolete as a result of new protocols and scientific needs. The biobankers seek to ensure the sustainable exploitation of the resource while respecting commitments made with participants and funding agencies.

The current section evaluates potential 'access strategies' employed by biobanks to manage access to their collections. A nonhierarchical list of various strategies can be found in table 1 . These strategies are not mutually ex- 
clusive and often interdependent in biobanks' access arrangements. They have been grouped into 4 categories of motivations. Furthermore, this section analyzes the effects of these strategies from both the biobank and the researcher's perspectives, with special attention given to strategies that could pose barriers to access. At this time, it is not the authors' intention to recommend or favor specific strategies over others. Rather, the intention is to present a descriptive account in order to guide biobankers in selecting strategies and devising access arrangements that address their specific needs and priorities.

\section{Promoting Targeted Objectives}

Each biobank is created to achieve a specific objective: perhaps a general goal of benefiting society or a particular community (e.g. disease-specific, institutional, national) [15] or contributing to public health in general. Having a specific objective is essential in convincing both the population and the funding agencies to participate in the biobank's project.

In order for a biobank to deliver on its anticipated social benefits, and for access arrangements to respect the biobank's early commitments, biobankers must be painstaking in their selection of evaluation criteria that will guide its review of access requests. A consideration of these evaluation criteria, in turn, provides insight into the nature of the biobank. For example, the Norwegian Institute of Public Health specifies that 'projects must contribute to scientific development in the research environment in Norway' [16]. In the same vein, some biobanks require that the project promotes their 'community commitment', with a strong emphasis on collaboration. For example, the Banco de ADN in Spain specifies that 'samples may be requested by any national, European or international research group working on cooperative projects in which Spain has an active part' [17]. This strategy is also used by the Danish National Biobank (DNB), which states that ' $\mathrm{t}$ ] he cohort is mainly financed by means of Danish national sources. It is [hoped] that epidemiologists from outside the country who wish to use data from the DNB cohort will do so in collaboration with Danish scientists' [18].

'Conditions for use' are the post-access counterpart of the aforementioned evaluation criteria and, similarly, provide insight into the nature of the biobank, its targeted objectives and initial commitments to funders and participants. Conditions for use qualify the permission for access granted to researchers and are generally expressed as restrictions that define and limit the scope of access. An example of a condition for use comes from the access policy of the National Heart, Lung and Blood Institute [19], which states that access can be revoked if samples are used for commercial research purposes, unless specific consent by sample donors has been obtained in advance.

Preferential Access. Another strategy observed seeks to encourage the financial or social involvement of a particular group or community. Some biobanks, for example, offer lower costs and/or more lenient procedures for targeted institutional researchers. These 'differential access procedures' reward local and institutional involvement in the creation of a biobank by providing preferential access terms on the basis of community or institutional membership.

Preferential access reflects an initial commitment by the biobank to the community. This targeted objective serves to generate scientific and economic benefits to the population that has invested public funds, samples and data in the infrastructure. In addition, it also seems to recognize and reward the direct involvement of people dedicated to building the project within the institution.

These diverse strategies, and the degrees to which they are implemented, can either reap great local benefits and generate international collaboration or dissuade researchers from collaboration and lower the biobank's appeal because of a perceived 'protectionist' approach. Building on the view that science is enriched by diversity in study designs [20], limiting access by external investigators could result in a loss of expertise and potential benefits for society.

\section{Promoting and Protecting the Biobank}

Biobanks are designed and built with the intention of being long-term research infrastructures, although public funding agencies might expect biobanks to become financially autonomous shortly after operations begin. To sustain their viability and longevity, biobanks rely in great part on the added value contributed by their users, as a means of enriching and consolidating their collection of data. Some strategies employed to promote and protect the resource include (1) requiring that researchers report results back to the biobank and (2) setting in place procedures that manage publications and acknowledgement of the biobank and/or its collection. These strategies are primarily intended to preserve the biobank's collection while attracting new financial and/or scientific commitments.

Reporting results back to the biobank 'valorizes' the resource. Biobanks might require the return of both positive and negative results (e.g. UK Biobank [21]), the re- 
turn of results as a supplement to the initial data (e.g. Estonian Genome Project [22]) or a periodic update on research results (e.g. every 6 months for ALSPAC [23]).

Publications and acknowledgment procedures are intended to increase the biobank's international exposure within the scientific community. An example is the GAZEL project which requires that all publications that benefited from access to the GAZEL collection must contain 'GAZEL' in the article's title [24]. These procedures are also intended to ensure easy reference on archive websites like PubMed. Many projects have also developed checklists for authorship designed to ensure that the biobank, the principle investigators and the funders are properly recognized.

To protect their reputation and avoid potential conflicts that might implicate the biobank in long and costly legal processes, biobankers will often include clauses that limit their responsibilities and legal liability and/or ensure arbitration in the event that a legal dispute arises.

Deadlines and Competition. Certain strategies also have the indirect effect of strict deadlines to an already competitive field. This is exemplified by the process of prepublication approval. For example, Generation Scotland states: 'All publications (e.g. submissions to journals and presentations) emanating from the Generation Scotland Project or Future Projects have to be sent to the Generation Scotland Research Proposal and Publications Committee for approval. We expect to process all papers within 30 days after the Research Proposal and Publications Committee at which they are discussed. We read all papers to check confidentiality is protected; to assist in the identification of patentable results; to ensure that the paper will not bring the Project or Future Projects into disrepute; to try to identify overlap with other papers published or in preparation. We also provide advice and feedback to authors where we feel this may be helpful' [25].

This comprehensive condition aims to protect participant confidentiality, the biobank's reputation and its trust relationship with the public and reduce overlap in scientific investigations. At first glance, such expectations may not seem to impose onerous obligations. However, in the context of intense competition and limited resources, these conditions can also impose tight deadlines on researchers during the feverish prepublication period and potentially expose their research to competitors before it is ready to be shared.

\section{Ensuring the Highest Standard of Research}

Biobanks are driven to develop their access arrangements as a means of ensuring the data will be used in high-quality research. Authenticating the qualifications of a researcher is crucial to safeguarding the integrity of all stakeholders and an obligation on the part of a publicly funded biobank. Failure by the biobank to verify the professional credentials of the researcher would constitute a breach of trust for participants. To meet this obligation, a biobank might require that researchers seeking access demonstrate their scientific competence, which is measured against a 'scientific merit' criterion (e.g. Singapore Tissue Network) as well as provide full references [26]. Another way biobanks enforce rigorous checks is through their own evaluation committees, comprised of scientific experts.

Another measure used by biobanks to ensure accountability regarding research projects which utilize their collection is the requirement of periodic reporting. Depending on the biobank, the delivery of these reports might be expected periodically or at the end of the project. Surveillance mechanisms may also be used to ensure security and confidentiality of the information and to deter misuse. For example, the CARTaGENE policy states that '[a]pproved users accessing the CARTaGENE resource shall agree to be audited for security and confidentiality of samples/data purposes. [...] These audits may be conducted without prior notice. The costs associated with these audits will be supported by CARTaGENE' [27].

In addition, special mechanisms have also been designed to deter researchers from breaching the conditions for use set by the biobanks and to ensure compliance with all ethical, legal and administrative requirements. In many cases, the principal investigator's signature on the contracts is considered binding for all staff who may come into contact with the data received. Signing confidentiality agreements before transferring data (e.g. NCI [28]) is one way of achieving this objective. For instance, the Joondalup Family Health Study states, 'If [researchers] do not comply with the agreement, the JFHS reserves the right to impose heavy penalties on the researcher, permanently bar them from any access to the database, have their ethics approval withdrawn (and their project then terminated), and/or report their conduct to their home institution or employer' [29]. These penalties supplement the ordinary legal remedies available.

Oversight and Administrative Demands. From the perspective of a bona fide researcher, oversight mechanisms may have the added benefit of protecting a researcher against doubts that might be raised regarding the scientific or ethical validity of his or her project or conduct. However, periodic reports that researchers are required to submit to ensure compliance with access conditions 
can be quite time-consuming. Too often the administrative workload attached to the oversight process creates delays that researchers wish to avoid [30].

\section{Supporting Access}

The motivations and guiding principles for biobanks described in the previous sections are not strictly tied to access per se; they also address other objectives of protecting and promoting of the biobank. However, many biobankers also seek to facilitate access to their biobank and promote the use of its contents. One of the strategies observed is opening access to the largest pool of researchers possible. For example, the CARTaGENE project allows access to 'national and international investigators involved in the public and/or private sectors [...] for scientific and academic research and public health research' [31]. In addition, the 'customer service' approach described in the first section of this paper is conducive to facilitating access, as is keeping access costs to a minimum (although most of the websites do not specify access fees).

Appropriate Access Fees. Establishing low access fees can be beneficial for both the biobank and researchers. By creating an affordable fee structure, biobanks can address several important issues, such as ease of access to their collection by researchers and the potential to generate benefits for the broader society (thus ensuring the biobank's relevance).

However, biobanks are faced with the challenge of how to remain financially viable, as they are not set up to be profit generators. Additionally, their launch and operation costs are variable, and access requests may vary in terms of the costs associated to fulfill them. Therefore, biobanks must find a balance between meeting their overhead costs and not charging prohibitive access fees. To address this challenge, biobanks are implementing business plans that amortize costs over the anticipated number of access requests by using a minimum accessfee structure.

The issue of access fees is an ongoing challenge because of the difficulties involved in determining the costs associated with such an unusual resource. The experience of CARTaGENE described in the following section demonstrates these challenges.

\section{Guiding Researchers to Biobank Access}

This section uses the example of CARTaGENE, a biobank based in Montreal, Quebec (Canada), to illustrate the challenges faced in the development of access ar- rangements. This illustration also highlights certain aspects related to access that may not be evident from the information available on biobank websites.

\section{The CARTaGENE Experience}

In short, CARTaGENE is a publicly funded project that aims to build a research infrastructure for population genomics research in Quebec. 'CARTaGENE seeks to create a resource for the advancement of genetic research, with the aim of improving the health of Quebecers. This public resource will operate under a governance framework and will consist of a databank and a biobank. CARTaGENE will contain environmental, demographic and health data. Its biobank will contain DNA, blood and urine samples' [31].

CARTaGENE began recruitment of participants in July 2009 and, as of October 2009, has completed close to 500 interviews. Its plan is to complete the recruitment phase by October 2010.

CARTaGENE is also part of a national biobank endeavor: The Canadian Partnership for the Tomorrow Project (CPTP) [32]. The objective of CPTP is to build 5 provincial cohorts that will form a national research platform. This platform aims to have 300,000 Canadians, including the 20,000 participants from CARTaGENE.

Even though this biobank was initially conceived for genetic and population genomics research purposes, it is now considered a useful research infrastructure for a much larger field of biomedical and social sciences, including, for example, demographic, sociology and ethics studies.

An independent Sample and Data Access Committee (SDAC) is in place to evaluate access demands and compatibility with CARTaGENE's objectives. The committee, in collaboration with the scientific management of CARTaGENE, will also define what results ought to be returned to the biobank. The SDAC has its own governance rules. At CARTaGENE, the governance structure imposed by institutional, social and legal constraints has resulted in a complex web of oversight bodies. The objective here is to examine the policy decisions behind its access strategy.

Supporting Access: Dealing with Intellectual Property (IP). In biobanks, there are 2 sources of IP rights. The first source originates from the creation of the biobank itself. Examples may include IP from the development of new software intended for use in conducting interviews with participants, copyrights in health questionnaires, patents in innovative equipment developed for the storage of samples, etc. 
The focus of this section is on the second source of IP, namely the IP developed as a result of access to data and material contained within the biobank. Such IP may include diagnostic tests, new applications for existing medications, new medications, etc. IP rights derived from research conducted with biobank data or samples should be clearly articulated prior to granting access to biobank materials to avoid potential misunderstandings and litigation.

Usually, when research materials are transferred between researchers, it is customary to address potential IP rights that might arise from collaboration through an MTA. These agreements either divide the arising IP according to the innovative addition made by the recipient of the material, or the parties agree to negotiate in good faith after an innovation is made according to the innovative input of each researcher.

Similarly, IP rights should be addressed where data are transferred to third parties. In the case of CARTaGENE, following a rigorous cost/benefit analysis on sharing IP developed by users, it was decided that the biobank (its host institution and/or its funders) would not own any part of IP rights developed by a researcher accessing CARTaGENE materials. Five reasons were given for this decision.

First, CARTaGENE is a publicly funded not-for-profit enterprise that does not incorporate royalties or license fees from IP in its business plan. CARTaGENE's goal is to provide access and recover costs related to that access in the form of access fees. Furthermore, it was determined that a business plan based on the recovery of potential and/or unpredictable royalties and license fees was unreliable.

Second, most investigators involved in biomedical research already have pre-existing IP arrangements with their institutions and/or other colleagues. In addition, large research projects often involve many investigators from various institutions further complicating IP issues. With so many stakeholders, such scenarios would result in complex and onerous IP negotiations for large research projects.

Third, one of CARTaGENE's fundamental objectives is the promotion of access and use of its infrastructure as a public resource. Therefore, the biobank determined that excluding itself from the fruits of IP developed through the use of its materials would make it attractive to potential investigators, especially for researchers from the private sector. Also, this approach was believed to be appealing because it reduces negotiation time between parties.

Promoting Access to Biobanks
Fourth, CARTaGENE reviewed other institutional IP agreements, the resources required to draft these agreements, the potential benefits that could be derived and the hindrances they could pose on free and open exchange of material/results. CARTaGENE felt that sharing in the IP rights could not conclusively yield an overall positive outcome $[33,34]$. Given that only a small percentage of such agreements actually yield any sort of financial benefit to the dispensing institution [30], and in light of the efforts and resources required to develop, administer and monitor IP agreements, CARTaGENE considered the exercise unproductive.

Fifth, CARTaGENE considered it ethically dubious to obtain rights to an innovation in which the biobank took no part in the inventive steps. While CARTaGENE's role as material supplier could be regarded as contributing to the development of new knowledge, knowledge alone cannot be considered a source of property rights. Rather it is the application of new knowledge in newly designed diagnostic tools, devices or drugs which is recognized as IP and therefore eligible for protection. Unless CARTaGENE is to take part in these practical applications, it is questionable whether it should be involved in any IP.

Unfortunately it is impossible to compare and contrast the rationale for CARTaGENE's IP policy with those of the aforementioned biobanks that were the source of this study, given that much of this information is not publicly available. However, it is hoped that the discussion of the reasoning behind CARTaGENE's IP policy will help other biobanks in devising or improving their own IP policies.

Promoting the Biobank: The Return of Results. As mentioned earlier in this paper, most biobanks, CARTaGENE included, are committed to promoting their services and resources by improving the biobank's content through research studies conducted using its data. To achieve this, investigators who access CARTaGENE resources are requested to participate in improving the resource by returning predetermined laboratory test results of transferred samples and other analytical results associated with coded participants back to CARTaGENE within a certain timeframe of accessing the material. The rationale for this return policy is the depletable nature of samples. Even though CARTaGENE's information brochure and consent forms allow for future recontact in order to update the collected data and obtain additional samples, these recontacts are expensive and time-consuming. Accordingly, there is a strong incentive to share the data produced as repeating identical manipulations on samples would waste data, samples and research funds.

Public Health Genomics 2011;14:104-114 
This led CARTaGENE to consider 2 secondary issues. First, to ensure the utility of results returned to the biobank, the analysis and/or manipulations of samples should provide high-quality results that are acceptable. Thus, an appropriate level of attention should be dedicated by the SDAC and CARTaGENE to ensure the scientific quality of results to be returned. Second, CARTaGENE should implement protocols to maximize the use of available samples. The following are ways in which this could be done: (1) releasing one sample for several research protocols where the analyses can be complementary; (2) funding additional analyses not included in the approved protocol; (3) encouraging researchers to include additional analyses within their protocol; (4) encouraging collaboration among researchers to ensure an optimal use of data and samples; (5) approving protocols that optimize use of samples.

The exact nature of results to be returned will be determined by the SDAC and the Scientific Director. Initially, what is contemplated is the return of genetic screening tests, genome-wide scans and, eventually, full sequences. To this might be added further biochemical and hematological analyses.

Even though such a policy might seem to add additional burdens to researchers, it was considered a reasonable compromise for a publicly funded biobank which does not benefit directly from IP developed by its resource users. In addition, it enables the reconciliation of 'open science' ideology with the sustainable development of the biobank.

Supporting Access: Establishing Appropriate Access Costs. Costs associated with access continue to be a challenging issue for CARTaGENE. One of CARTaGENE's objectives is self-sustainability through access fees throughout its life cycle (expected to be 50 years). In addition, it is largely settled that CARTaGENE is a not-forprofit enterprise seeking above all else to stimulate research in the field of genomics. Given CARTaGENE's priorities, a differential cost scheme for public, private and international researchers appeared to be an appropriate strategy.

Two types of access fees were established: a flat rate access fee and a variable sample access fee. The first covers the cost of evaluating the project by SDAC and access to data (regardless of the amount of data). The variable sample access fee is charged for extraction of samples from the biobank and their manipulation. This fee varies according to the number of samples extracted. It was believed that this approach would be the most predictable and straightforward way to ensure the minimal recovery of costs. Transportation fees for samples are billed separately to investigators and institutions.

However, the issue of costs is still being worked out internally. The biobank's investigators and management are aware that imposing excessive costs could be a hurdle to access. Thus, they intend to find ways to build in measures that will ensure the optimal use of CARTaGENE while securing a necessary level of sustainability.

Supporting Access: Granting Exclusive Access? Although exclusive access rights to CARTaGENE data are not granted to investigators, for each approved protocol CARTaGENE's Data Curator will create subsets of data from the raw data, using predetermined parameters and criteria, as a means of supporting access and ensuring optimal use of the database. These parameters and criteria are determined by the vetted investigator and in collaboration with CARTaGENE. The data subset and the parameters according to which it was created are unique to each study and are not shared with a third party without the prior consent of the researcher. In fact, only certain information about approved studies, which are specified in the access policy, can be made public.

It is possible that more than one independent researcher might present similar studies in which almost identical data could be required. In the case where only data is requested, CARTaGENE is not concerned as a certain level of redundancy in science is welcome as a means of validating results and conclusions. However, things get a bit more complicated in cases where depletable samples are involved. In such situations, the SDAC has discretion in deciding whether redundancy is wasteful and may even suggest collaboration between investigators.

\section{The Contribution of $P^{3} G$}

International scientific collaboration has become fundamental to the success of complex research projects, especially in the study of genetics and environmental factors. However, the diversity of access rules and procedures could create a complex web negatively affecting international collaboration.

Recognizing the concrete needs of biobankers and researchers, $P^{3} \mathrm{G}$ develops research tools for public population-based biobanks and facilitates harmonization and collaborations between biobanks. The tools are developed by drawing on the expertise and experience of biobankers from around the world. This paper intends to serve as a first step towards proposing generic access agreement clauses that could be adapted by various biobanks according to their local needs and requirements. 
This paper, and the comparative chart which preceded it [35], are part of an initiative to articulate the core elements of access arrangements, so as to encourage biobankers to address the numerous issues that are relevant to access. This initiative could eventually lead to a harmonized approach to access arrangements, while allowing for enough flexibility to include context-specific adjustments. In collaboration with the $\mathrm{P}^{3} \mathrm{G}$ Ethics and Governance Working Group, some results were presented in Philadelphia in November 2008, and follow-up discussions are planned for the international meetings scheduled in 2009. Based on these discussions, core elements of data access arrangements have been identified and listed in: ' ${ }^{3} \mathrm{G}$ Sample and Data Access: Core Elements' [35]. This document, developed by the Ethics and Policy Making Core at the Centre de recherché en droit public, identifies core elements of the Sample and Data Access Policies of $\mathrm{P}^{3} \mathrm{G}$ member biobanks. An analytical paper by $\mathrm{P}^{3} \mathrm{G}$ 's Ethics and Policy Making Core can also be found on the $\mathrm{P}^{3} \mathrm{G}$ Observatory [35]. This tool provides core issues that should be considered when drafting an access agreement for samples and/or data of a biobank and suggests a generic clause for each core issue identified.

As collaboration is a valuable means of communication and exchange, this comparative and analytic study is particularly relevant to international networking. Addressing access arrangements is a necessary step in finding better ways to harmonize biobanks. When interoperationalization is sought, specific restrictions concealed in variable policies, procedures and contracts constitute an important hurdle. $\mathrm{P}^{3} \mathrm{G}$ intends to move forward in establishing tools for collaboration and finding solutions to facilitate access to biobanks and the free flow of data for research purposes.

\section{Conclusion}

In these early stages, access arrangements for biobanks should be regarded as work in progress. This paper, the first to attempt to articulate the complexities of access arrangements, presents a snapshot of the current approaches to access and the challenges to and diversity of these approaches. The paper also provides insight into the values, motivations and priorities of a biobank. Further research in the area can eventually lead to recommendations on how biobankers could position themselves to be on the 'right side' of the line between facilitating and hindering collaboration, while still attending to the motivations that were behind the biobank's creation.
The relevance of a biobank and its success will be ultimately determined by how scientific communities, local and international, will take advantage of their resources and services. Accordingly, access arrangements must be worded clearly and facilitate the use of data. As public funds are used to develop national biobanks, it is important that beneficiary biobanks satisfy their funding agency's expectations and contributions toward the betterment of society. One might ask whether it is ethical to use consent forms and public outreach tools to identify the potential benefits of biobanks when the resource is underused due to access barriers and may not deliver the intended benefits. These concerns were central to the development of CARTaGENE's - and other biobanks' access policies as was striking a balance between the rights and obligations of the various stakeholders involved.

Along with the consent form, access arrangements are very much in the public domain. They send a very visible message about the biobank's level of openness and potential for collaboration. Thus, each biobank ought to determine its foundational values and ensure they are properly reflected through its access arrangements.

\section{Acknowledgments}

Sabrina Fortin is the recipient of a Canadian Institute of Health Research Doctoral Grant. The author recognizes the financial support of Apogée-Net, a network to assist policy-making in genetics, which enabled an internship within the $P^{3} G$ Secretariat. $\mathrm{P}^{3} \mathrm{G}$ Consortium is a project financed by Genome Canada and Genome Québec. Saminda Pathmasiri recognizes the financial contribution of the Canadian Institute of Health Research and the support of his thesis director, Professor Bartha Maria Knoppers.

\section{References}

$1 \mathrm{P}^{3} \mathrm{G}$ Observatory: Study catalogue. 2009. Available at http://www.p3gobservatory.org/ studylist.htm.

2 OECD: Principles and Guidelines for Access to Research Data from Public Funding. 2007. Available at http://www.oecd.org/dataoecd/ 9/61/38500813.pdf.

$\checkmark 3$ Cambon-Thomsen A, Rial-Sebbag E, Knoppers BM: Trends in ethical and legal frameworks for the use of human biobanks. Eur Respir J 2007;30:373-382.

4 Zika E, Schulte in den Bäumen T, Kaye J, Brand A, Ibarreta D: Sample, data use and protection in biobanking in Europe: legal issues. Pharmacogenomics 2008;9:773-781. 
5 Human Genome Project Information: Policies on Release of Human Genomic Sequence Data. 1996. Available at http://www. ornl.gov/sci/techresources/Human_Genome/research/bermuda.shtml.

6 Wellcome Trust: Sharing Data from Largescale Biological Research Project: A System of Tripartite Responsibility. 2003. Available at http://www.genome.gov/Pages/Research/ WellcomeReport0303.pdf.

7 Pennisi E: Data Sharing. Group calls for rapid release of more genomics data. Science 2009;324:1000-1001.

8 Wellcome Trust: Policy on Data Management and Sharing. 2007. Available at http:// www.wellcome.ac.uk/About-us/Policy/Policy-and-position-statements/WTX035043. htm.

9 Medical Research Council: Policy on Data Sharing and Preservation. 2007. Available at http://www.mrc.ac.uk/Test/PolicyGuidance_old/EthicsAndGovernance/ DataSharing/Current Actitivies/ MRC002553\#P17_947.

10 Genome Canada: Data Release \& Resource Sharing Policy. 2005. Available at http:// www.genomecanada.ca/medias/PDF/EN/ DataReleaseandResourceSharingPolicy.pdf.

11 Avon Longitudinal Study of Parents and Children (ALSPAC): Material Service Level Agreement. Available at http://www.bristol. ac.uk/alspac/documents/appendix8-material-sla.pdf, ALSPAC: Genetic Data Service Level Agreement. Available at http://www. bristol.ac.uk/alspac/documents/appendix8a-dsla.pdf.

12 National Institutes of Health: Cooperative Agreement. Available at http://www.ott.nih. gov/forms_model_agreements/.

13 The Twin Research and Epidemiology Unit (TRU): Policy Document for Collaboration. Available at http://www.twinsuk.ac.uk/collaborations/TRU_Policy_Collaborations. pdf.
14 Cancer Care Ontario: iPort ${ }^{\mathrm{TM}}$ Access Request Form. Available at https://webcon.cancercare.on.ca/iPort_reg/.

15 Brunger F: Problematizing the notion of community in research ethics; in Knoppers BM (ed): Populations and Genetics - Legal and Socio-Ethical Perspectives. Leiden, Martinus Nijhoff Publishers, 2003.

16 Norwegian Institute of Public Health: Which additional conditions apply to biological material? Available at http://www.fhi.no/eway/ default.aspx? pid $=238 \& \operatorname{trg}=$ Area_5954\& MainArea_5811=5853:0:15,3894:1:0:0:::0: 0\&MainLeft_5853=5954:0:15,3894:1:0:0:::0: 0\&Area_5954=5825:69864::1:5956:1:::0:0.

17 Banco Nacional de AND: Who can request samples. Available at http://www.bancoadn. org/en/muestras2.htm.

18 Danish National Birth Cohort: Conditions for access to data. Available at http://www. serum.dk/sw42351.asp.

19 National Heart Lung and Blood Institute: Information for researchers. Available at http:// www.nhlbi.nih.gov/resources/deca/policy_ new.htm\#i.

20 Hudson T, quoted in Trivedi B: Biomedical science: betting the bank. Nature 2008;452: 926-929.

21 UK Biobank: Ethics and Governance Framework. Available at http://www.ukbiobank. ac.uk/docs/EGF20082.pdf.

22 Estonian Genome Project: University of Tartu Makes Data Available for Research. Available at http://www.geenivaramu.ee/index. php?id=111.

23 ALSPAC: Material Transfer Agreement Available at http://www.bristol.ac.uk/alspac/documents/appendix7-mta-2.pdf.

24 Inserm: Cohorte GAZEL: Règles de fonctionnement de la cohorte Gazel. http://www. gazel.inserm.fr/pdf/REGLEMENTGAZEL. pdf.
25 Generation Scotland: Authorship, Publications and Papers Checklist (appendix 13). Available at http://www.generationscotland. org/Documents/GSMAPAppendix13v3Mar08.pdf.

26 Singapore Tissue Network: Providing Tissues to the Scientific Community. Available at http://www.stn.org.sg/services_researchcomm.htm.

27 CARTaGENE: Sample and Data Access Policy. Draft December 2008

28 National Cancer Institute: Confidential Disclosure Agreement. Available at http://ttc. nci.nih.gov/forms/1WAYOut-CDAFinal11-01.pdf.

29 Joondalup Family Heath Study: FAQ. Available at http://www.jfhs.org.au/uploads/yO/ Ks/yOKsxBabfhrMOLRCPq2j8g/JFHSFAQ-ELSI.pdf.

30 Fortin S, Knoppers BM: Secondary Uses of Personal Data for Population Research. Genomics Soc Policy 2009;5:80-99.

31 CARTaGENE: What is CARTaGENE? 2008. Available at http://www.cartagene.qc.ca/index.php?option $=$ com_content $\&$ task $=v i e w \&$ id $=1 \&$ itemid $=522 \&$ lang=english .

32 Canadian Partnership against Cancer. 2008. Available at http://www.partnershipagainstcancer.ca/tomorrow_update.

33 Statistic Canada: Survey of Intellectual Property Commercialization in the Higher Education Sector. 2006 and 2005 published in 2008. Available at http://www.statcan. gc.ca/pub/88-222-x/88-222-x2008000-eng. pdf.

34 Lei Z, Juneja R, Wright BD: Patents versus patenting: implication of intellectual property protection for biological research. Nat Biotechnol 2009;27:504-505.

$35 \mathrm{P}^{3} \mathrm{G}$ Observatory: Ethics, Governance and Public Engagement. 2009. Available at http:// www.p3gobservatory.org/repository/ethics. htm. 